\title{
Imaging the two wind post-AGB interaction in M 1-92
}

\author{
J. Alcolea and V. Bujarrabal \\ Observatorio Astronómico Nacional, Apdo. 1143, E-28800 Alcalá de \\ Henares, Spain
}

\begin{abstract}
M 1-92, Minkowski's Footprint, is a well studied protoplanetary nebula (PPN). Here we present high resolution observations of this object in atomic and molecular lines, as well as in the continuum, at optical and radio wavelengths. From these observations we conclude that the present bipolar nebula is the old AGB shell, strongly modified by the interaction with some powerful post-AGB bipolar ejections, which started suddenly about 900 years ago. The nature of these post-AGB ejections as well as its comparison with the present bipolar fast flow are discussed. We conclude that a mechanism different from photon pressure must be invoked in order to explain the morphology and dynamics of M 1-92 and other similar objects.
\end{abstract}

\section{Introduction}

One of the crucial phases of the stellar evolution that are still not well known is the post-AGB evolution of late-type stars toward the Planetary Nebula (PN) stage. Today we know that the evolution of both the central star and its surrounding envelope is driven by the processes of copious mass loss occurring at the very end of the AGB phase. In a few thousand years the central star becomes a hot white dwarf, ionizing the AGB shell which then shines as a PN. This evolution is accompanied by drastic changes in the chemistry, shape and kinematics of the circumstellar envelopes. We note in particular the break-up of the spherical symmetry characteristic of AGB circumstellar shells (Neri et al. 1998 ), that is replaced in many post-AGB objects by a clear bipolar appearance.

For a better understanding of this process, we must study in detail the few objects that are suffering these deep transformations right now, i.e. the objects in the transition phase, usually known as protoplanetary nebulae (PPNe). Here we present a summary of our results on one of these few objects, M 1-92, Minkowski's Footprint (see also the contribution by Sánchez Contreras et al. in this volume). These results, which have been very fruitful in part because of the explicitness of the object, have allowed us to draw a global but also very detailed picture of the post-AGB processes in this source.

M 1-92 is a typical bipolar reflection nebula, $10^{\prime \prime}$ long, with a clear symmetry axis oriented in the sky nearly in the NW-SE direction (Minkowski 1946, Herbig 1975, see Fig. 1c). The two lobes are separated by an equatorial disk of dust, partially obscuring the South lobe (the farthest one) and the central star. For a distance of $3 \mathrm{kpc}$, the total luminosity of M1-92 is about $10^{4} L_{\odot}$ (Calvet \& 
Cohen 1978), and from the star light scattered by the dust grains, a temperature of $20000 \mathrm{~K}$ has been derived for the central source (Herbig 1975; Cohen \& Kuhi 1977). Some authors claim that this central source is in fact a binary system in view of its composite spectrum (Feibelman \& Bruhweiler 1990), however no direct observation of the companion exists. M 1-92 presents many signs of being a relatively young PPNe, and therefore it constitutes a very good candidate for studying the post-AGB processes when they are occurring or are still very recent. We have conducted on this source a series of high-resolution observations at optical and radio wavelengths, in order to probe the different components of the circumstellar envelope.

\section{Molecular line observations}

The observations of the molecular envelope of M 1-92 were conducted using the IRAM Plateau de Bure interferometer, from 1993 to 1997 . We observed the ${ }^{12} \mathrm{CO}$ and ${ }^{13} \mathrm{CO} J=1-0$ lines with a spatial resolution of $\sim 2$, and the ${ }^{13} \mathrm{CO} J=2-1$ line with $1^{\prime \prime}$ resolution. See Bujarrabal et al. (1994, 1997 and 1998b) for more details. From these $\mathrm{CO}$ observations we have been able to derive a very detailed view of the structure and kinematics of the neutral envelope, which consists in three different components: an equatorial disk, dividing two elongated emptied shells (each one spatially coincident with each lobe of the nebula), ended in two high velocity knots expanding along the symmetry axis at a deprojected velocity of $70 \mathrm{~km} \mathrm{~s}^{-1}$ (Fig. 1a-b, see also Figures 1 and 2 in Bujarrabal et al. 1998b). The total size of the nebula is $4^{\prime \prime} \times 11^{\prime \prime}\left(1.5 \times 510^{17} \mathrm{~cm}\right.$ deprojected $)$, to be compared with the small width of the walls of the emptied shells, of only $0^{\prime \prime} 6\left(210^{16} \mathrm{~cm}\right)$. This width is also comparable to the thickness of the equatorial disk and to the size of the high velocity knots, i.e. the nebula is relatively large but consists of several very thin sheet-like components.

From a model fitting of our maps obtained in the three CO lines, we have estimated the main physical parameters of the envelope, under the assumption that all three lines are thermalized and adopting a ${ }^{13} \mathrm{CO} / \mathrm{H}_{2}$ relative abundance ratio of $210^{-5}$. We have obtained that the density gradually decreases from $310^{5} \mathrm{~cm}^{-3}$, at the equatorial plane, to $510^{4} \mathrm{~cm}^{-3}$, at both high-velocity tips. The kinetic temperature is very low, $15 \mathrm{~K}$ in the central disk and only $10 \mathrm{~K}$ in the outer parts of the nebula. We also derive a total mass of $0.9 M_{\odot}$, this high value being quite comparable to the expected total mass lost during the AGB-phase. The kinematics of the whole molecular envelope is dominated by a strong and nearly constant velocity gradient along the nebular axis, of $7 \mathrm{~km} \mathrm{~s}^{-1}$ per arcsec (deprojected), from which we derive a dynamical age of $900 \mathrm{yr}$ for the present $\mathrm{CO}$ kinematics. The mean gas expansion velocity in the lobes, about $40 \mathrm{~km} \mathrm{~s}^{-1}$, is found to be in agreement with the mean dust expansion velocity, obtained from the Doppler shift measurements of scattered stellar line emission (Solf 1994). The equatorial disk is found to be in expansion at a moderate (typical AGB) velocity of $\sim 8 \mathrm{~km} \mathrm{~s}^{-1}$; no sign of rotation around the symmetry axis is found.

In view of these results we conclude that this molecular envelope is the remnant of the AGB shell; a mass loss as copious as about $10^{-4} M_{\odot} \mathrm{yr}^{-1}$ at the end of the AGB is needed in order to account for the computed total mass. 
The structure and kinematics of the old AGB envelope would have been strongly modified as a result of a strong wind interaction which occurred some $900 \mathrm{yr}$ ago. The nearly constant velocity gradient suggests that, during most of that time, the acceleration has been negligible, i.e. the process responsible for the present dynamics probably lasted for less than or about $100 \mathrm{yr}$. (This Hubblelike velocity field is expected in the case of a sudden interaction.) Using this result, and thanks to the high quality of our interferometric maps, we have also been able to obtain a direct 3-D model of the nebula by simply assuming that the velocity field is proportional to the radial distance to the center of the nebula. For this simple velocity field, one can use the observed projected velocity shift to recover the position of the emitting gas along the line of sight (see Alcolea \& Bujarrabal 1999). Following this procedure we can visualize the structure of the envelope, i.e. the shape of the equatorial disk and of the $\mathrm{CO}$ walls, and project it onto the plane of the sky (see Fig. 1b). This image is very useful for comparisons with the other images taken for the nebula.

The shock interaction between the old AGB wind and some very powerful post-AGB bipolar ejection occurred via a momentum driven bow shock, after which a strong cooling has taken place (a quasi-isothermal shock). This would explain the present low kinetic temperature and highly ordered structure of the nebula. Most remarkable is the amount of axial (along the symmetry axis) momentum carried by the molecular envelope. Assuming an AGB radial expansion velocity of $8 \mathrm{~km} \mathrm{~s}^{-1}$ (i.e., assuming that the equatorial disk is indeed the only part of the nebula which has not been modified by the post-AGB interaction), we estimate that the linear momentum gained by the envelope as a result of the shock interaction is $310^{39} \mathrm{~g} \mathrm{~cm} \mathrm{~s}^{-1}$. This is about 1000 times the total mechanical momentum of the photons emitted by the central star during the time we have estimated the interaction lasted for. We must then conclude that photon pressure could not power the strong post-AGB ejections responsible for the present kinematics. This discrepancy still holds if we relax the interaction time up to the total post-AGB life of M 1-92 or if we consider multiple scattering (Bujarrabal et al. 1998b). Another mechanism, turned on right at the end of the AGB phase, must be invoked. This axial momentum excess is not exclusive of the neutral envelope of M 1-92, but on the contrary it is a relatively common feature of the envelopes around post-AGB sources (see also the contribution by Sánchez Contreras et al. in this volume). Although the nature of the mechanism responsible for the post-AGB dynamics is still unknown, the similarities between the post-AGB ejections and those from low mass young stellar objects, like for instance the momentum excess and high collimation (see next section), suggest that a similar process, ultimately powered by mass accretion, might be at work also at the beginning of the post-AGB era.

\section{HST-WFPC2 observations}

The present post-AGB ejections have been imaged through the observations of forbidden atomic lines using the WFPC2 of the HST. We obtained images of the SII and OI lines, two well known tracers of shocks, as well as of the OIII line to probe the excitation of the different regions. The continuum at $547 \mathrm{~nm}$ and the $\mathrm{H} \alpha$ line were also observed (Fig. 1c-d, Bujarrabal et al. 1998a). The results show 


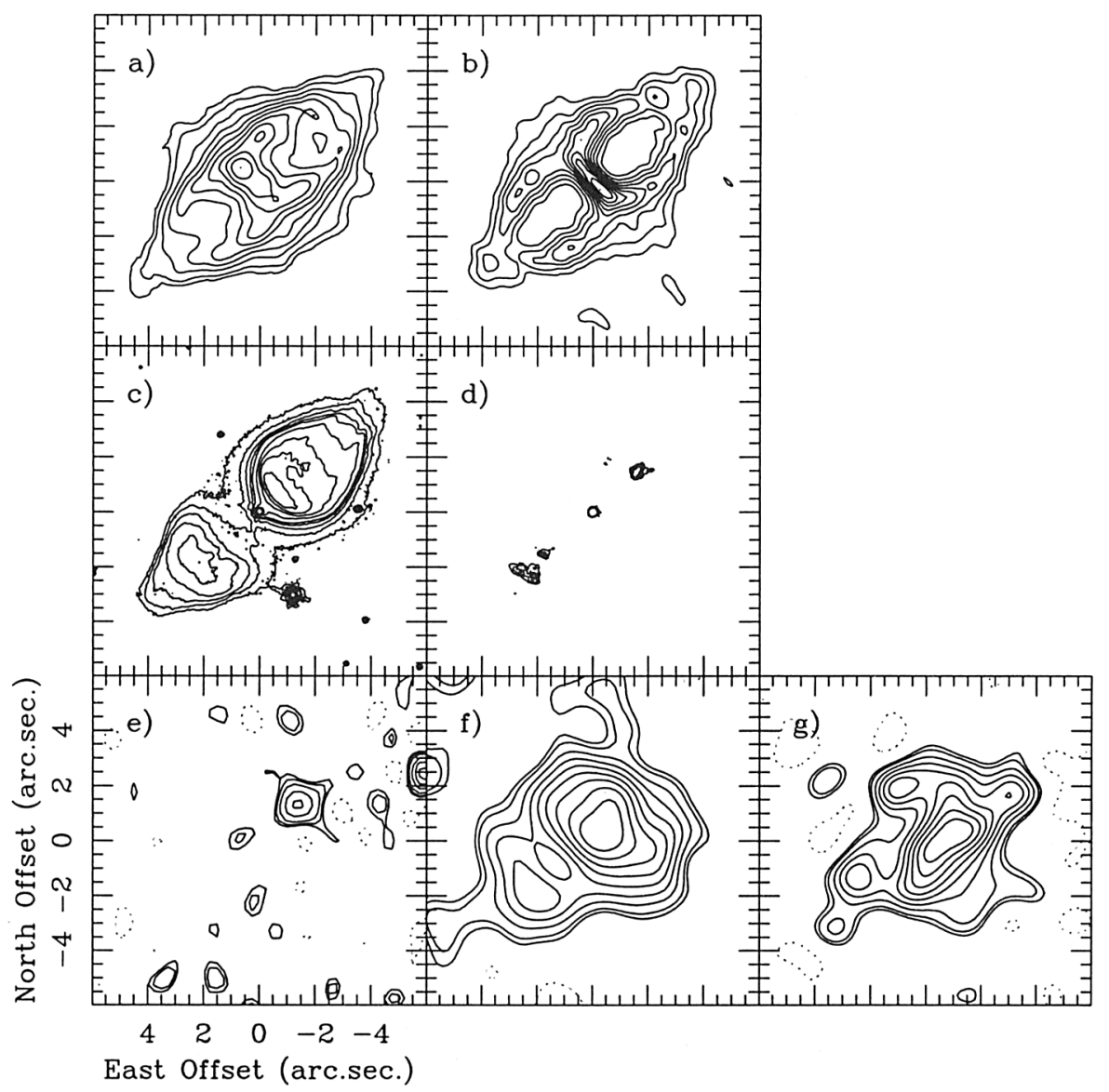

Figure 1. Different views of M 1-92. Dashed lines represent negative contours. Fig. 1a: the molecular shell of M 1-92 from the ${ }^{13} \mathrm{CO} J=2-$ 1 total integrated emission. Fig. 2b: structure of the molecular shell (the $\mathrm{CO}$ walls plus the equatorial disk) projected onto the plane of the sky, obtained after a $3-\mathrm{D}$ reconstruction of nebula from the ${ }^{13} \mathrm{CO}$ $J=2-1$ data. Fig. 1c: continuum image at $547 \mathrm{~nm}$. Fig. 1d: image of the shocked gas in M1-92 as seen in the SII forbidden line. Fig. 1e: continuum emission at $6 \mathrm{~cm}$. Fig. 1f: continuum emission at $2.6 \mathrm{~mm}$. Fig. 1g: continuum emission at $1.3 \mathrm{~mm}$. 
that the present bipolar post-AGB ejection is highly collimated. The emission in the SII and OI lines originates from a chain of compact knots along the axis of the nebula. Long slit observations of these lines (Solf 1994) show that this shocked atomic gas is expanding along the axis at mean velocities of about $90 \mathrm{~km} \mathrm{~s}^{-1}$, about twice the expansion velocity of $\mathrm{CO}$ at similar distances from the star. When comparing these observations with the structure of the CO shell (Figs. 1b and 1d), it is clearly seen that the optical emission can not be directly due to shocks between the faster bipolar outflow and the molecular shell. The $\mathrm{OI}$ and SII knots are located just in the middle of the $\mathrm{CO}$ cavities, therefore this emission must be mostly due to shocks taking place within the jet itself. Only in the South lobe a structure perpendicular to the nebular axis is detected, just at the inner boundary of the $\mathrm{CO}$ shell. This could be the Mach disk, the point where the fast jet and molecular shell collide today. The results from the OIII observations suggest that the excitation is higher in the North lobe. No sign of the leading bow shock, produced in the strong interaction responsible for the present kinematics, has been found. This shock, if still present, must be in front of the accelerated molecular gas, but remains undetected till now.

The present post-AGB ejection is not affecting any more the kinematics of the molecular shell: apparently the two-wind interaction now is much less energetic than it was when it strongly accelerated the nebular gas $900 \mathrm{yr}$ ago. For the ionized gas, we estimate a total axial momentum much lower, between $10^{37}$ and $10^{38} \mathrm{~g} \mathrm{~cm} \mathrm{~s}^{-1}$. We must therefore conclude that present post-AGB ejection is substantially different (at least much less energetic) from what it was before, although we can not rule out that the mechanisms producing both post-AGB flows could be similar.

\section{4. $\mathrm{cm}$ and $\mathrm{mm}$ continuum observations of $\mathrm{M}$ 1-92}

$6 \mathrm{~cm}(4.8 \mathrm{GHz})$ continuum observations of $\mathrm{M} 1-92$ were also performed, using the VLA interferometer in A configuration in October 1995, the resulting synthesized HPBW being 0.9. As it is shown in Fig. 1e the emission comes from two well separated spots of similar intensity located on the North-West side of the nebula. Both spots are unresolved by our beam. One of the spots is coincident with the high excitation knot seen in optical forbidden lines along the North side of the nebular axis. On the contrary, the other spot (just at the right border of the panel) originates from a position well outside M 1-92 and very probably is not associated to our source. Our map is compatible with the one previously obtained by Bowers \& Knapp (1989) in which, due to the lower resolution, both spots appeared as an East-West elongated structure at the North lobe of the nebula. Most remarkable is the good correspondence of the first spot (the only one related to $M$ 1-92) with the emission from shock excited atomic gas. The $6 \mathrm{~cm}$ continuum emission seems to be due to thermal free-free emission by electrons (no polarization has been detected). Since no dust neither gas opacities are expected at this low frequency, the fact that no $6 \mathrm{~cm}$ continuum emission is detected in the South lobe confirms that the present post-AGB bipolar ejections in $\mathrm{M} \mathrm{1-92}$ are not symmetric, in agreement with the results obtained from the OIII/OI line ratio. 
Simultaneously to our Plateau de Bure CO line observations, we have also mapped the continuum emission of M 1-92 at 2.6 and $1.3 \mathrm{~mm}$ (113 and 223 $\mathrm{GHz}$ respectively). As it is shown in Fig. $1 \mathrm{f}-\mathrm{g}$, the emission is resolved in the two bands, being elongated in the direction of the symmetry axis. The total detected emission is $16 \mathrm{mJy}$ at $2.6 \mathrm{~mm}$, and $74 \mathrm{mJy}$ at $1.3 \mathrm{~mm}$. From these values we derive a spectral index of 2.3 , fully compatible with optically thin dust emission. We also note that at $2.6 \mathrm{~mm}$, the location of the maximum is not coincident with the center of the nebula, it is slightly displaced to the North along the axis of symmetry. This can not be explained in terms of opacity, that must be very low also in this band. A possible explanation would be that at $2.6 \mathrm{~mm}$ there is still some contribution from the free-free emission detected at $6 \mathrm{~cm}$. This would be possible only if this free-free emission is due to an ionized jet (for which a spectral index of about 0.6 is expected) and does not come from a standard HII-like region.

Acknowledgments. This work has been partially supported by the Spanish DGES project PB96-0104.

\section{References}

Alcolea J., Bujarrabal V., 1999, in preparation

Bowers P.F., Knapp G.R., 1989, ApJ 347, 325

Bujarrabal V., Alcolea J., Neri R., Grewing M., 1994, ApJ 436, L169

Bujarrabal V., Alcolea J., Neri R., Grewing M., 1997, A\&A 320, 450

Bujarrabal V., Alcolea J., et al., 1998a, A\&A 331, 361

Bujarrabal V., Alcolea J., Neri R., 1998b, ApJ 504, 915

Calvet N., Cohen M., 1978, MNRAS 182, 687

Cohen M., Kuhi L.V., 1977, ApJ 213, 79

Feibelman W.A., Bruhweiler F.C., 1990, ApJ 354, 262

Herbig G.H., 1975, ApJ 200, 1

Minkowski R., 1946, PASP 58, 305

Neri R., Kahane C., Lucas R., Bujarrabal V., Loup C., 1998, A\&AS 130, 1

Solf J., 1994, A\&A 282, 567 